\title{
Evaluating outcomes in ultrasound-guided regional anesthesia
}

\author{
Ki Jinn Chin, MD $\cdot$ Vincent Chan, MD
}

Published online: 10 September 2009

(C) Canadian Anesthesiologists' Society 2009

Ultrasound imaging has revolutionized the practice of regional anesthesia. Perhaps the most remarkable aspect of this revolution is that it has led to many more anesthesiologists incorporating regional anesthesia into their practice. Within the last decade, ultrasound-guided regional anesthesia (UGRA) has evolved from an experimental technique practiced by experts and enthusiasts to an established, clinically relevant modality that has captured the attention of the general anesthesia community.

The literature on UGRA has similarly evolved in both volume and content. A PubMed search using the terms "ultrasound" and "nerve block" returned 32 citations for 2004, 82 citations for 2006, and 158 citations for 2008. Early publications were centered mostly on studies of basic peripheral nerve block techniques and the relevant ultrasonographic anatomy, and usually the techniques were performed in volunteers and small selected patient cohorts. We have now reached the stage where this knowledge base is largely established for the commonly performed blocks. Subsequent studies took the next logical step by comparing UGRA with the conventional or standard technique in use, in this case neurostimulation. Although the debate regarding the superiority of ultrasound guidance over neurostimulation remains unsettled, ${ }^{1}$ it is increasingly accepted that the ability with ultrasound to directly visualize the needle, the target nerve, and the local anesthetic deposition is a definite advantage. It is worth remembering that when neurostimulation was first introduced, a similar debate arose as to whether it was superior to the elicitation of paresthesia., ${ }^{2,3}$ Neurostimulation gradually became accepted as the

K. J. Chin, MD $(\bowtie) \cdot$ V. Chan, MD

Department of Anesthesia, Toronto Western Hospital, 399

Bathurst Street, McL 2-405, Toronto, ON M5T 2S8, Canada

e-mail: kijinn.chin@uhn.on.ca; gasgenie@yahoo.co.uk standard of practice, not because of large, good-quality randomized controlled trials (RCTs) demonstrating better outcomes but because practitioners recognized that an objective endpoint was more advantageous for adequate needle-nerve proximity than a subjective one.

It is time, therefore, to move beyond asking whether neurostimulation or ultrasound guidance should be used in performing regional anesthesia to asking how we can effectively incorporate UGRA into clinical practice. Unfortunately, obtaining answers to this question from the literature is not a straightforward affair. There are several confounding factors that must be considered when attempting to generalize the results of published studies to one's own practice.

\section{Operator experience}

A major confounder in interpreting studies of UGRA is operator training and experience. While ultrasound has demystified the art of regional anesthesia to a large extent, it is clear that there is still a learning curve for safe and effective performance of UGRA. ${ }^{4}$ To date, most of the published studies have been conducted by a few experienced practitioners, and often just one or two per study. While this is inevitable in the evolution of any new technique, the results of these studies may not be generalizable to the growing number of anesthesiologists who are just beginning to use UGRA. Future studies, especially comparative studies, must address the potential bias that results from operator experience with, or preference for, one technique over another. One solution is to compare two groups of experts, e.g., weigh one group that uses neurostimulation exclusively against another group experienced in ultrasound guidance. ${ }^{5}$ Another more feasible option is to study a large pool of 
inexperienced operators performing UGRA, such as a cohort of anesthesiology residents undergoing a rotation in regional anesthesia. In our opinion, studies that demonstrate the advantages of UGRA in residents, such as those by Orebaugh (improved ease of block performance) ${ }^{6}$ and Grau (shortened learning curve for epidural anesthesia), ${ }^{7}$ offer some of the most compelling evidence for the benefits of UGRA because they reflect the experience that novices are likely to face when incorporating UGRA into their clinical practice.

The American Society of Regional Anesthesia and the European Society of Regional Anesthesia and Pain Therapy recently published the first iteration of their joint recommendations for education and training in UGRA. ${ }^{8}$ Standardization of training will help address the issue of operator bias in future studies. However, much work still needs to be done in this area. The definition of competency is still open to debate, and researchers are only beginning to characterize the learning curves associated with various ultrasound-guided techniques. ${ }^{9}$ It should be recognized that different UGRA blocks have different levels of complexity and require different levels of skill. Optimal methods of instruction in the skills required for UGRA have also yet to be established and will no doubt evolve along with the ongoing development of more sophisticated models and simulators.

\section{Variations in technique}

When analyzing the literature on UGRA, it is important to appreciate the subtle differences in technique that may exist between studies and to question whether these may have influenced the results. For example, ultrasound imaging capability varies among machines and studies. Higher-resolution transducers and features, such as compound imaging, significantly improve tissue and needle visualization and may also improve block performance. Was an in-plane or out-of-plane needle approach used? What endpoints were utilized for needle-nerve contact and local anesthetic injection? This last question is of particular importance, as block efficacy is probably influenced by the site and pattern of local anesthetic deposition and the volume of anesthetic administered. Recent studies have explored the minimum effective volume for various ultrasound-guided blocks ${ }^{10,11}$; however, the endpoints for local anesthetic deposition that are associated with optimal results have yet to be determined. Given that nerve architecture varies from one anatomical site to another, it is likely that these endpoints will differ from block to block.

Some of these points can be illustrated by comparing three studies that weighed ultrasound-guided supraclavicular against infraclavicular block but arrived at very different results ${ }^{12-14}$ (Table 1). Arcand et al. ${ }^{12}$ reported a higher incidence of anesthesia or analgesia in the five terminal nerve distributions of the brachial plexus with the supraclavicular block; Koscielnak-Nielsen et al. ${ }^{13}$ found the converse to be true; and Tran et al. ${ }^{14}$ found no difference. A higher complication rate with the supraclavicular block was also seen in two studies ${ }^{13,14}$ but not in the third. ${ }^{12}$ Careful analysis of the studies suggests that the discrepancies can be explained by differences in study methodology (e.g., the number and experience of operators) and block technique. For example, the failure of Arcand et al. ${ }^{12}$ to specify a U-shaped postero-latero-medial distribution of local anesthetic as an endpoint for infraclavicular blockade may have contributed to the lower success rate observed. ${ }^{15}$ Similarly, the optimal technique of supraclavicular blockade as used by Tran et al. ${ }^{14}$ may involve injection of local anesthetic deep to the plexus at the junction of the first rib and subclavian artery ${ }^{16,17}$ rather than superficial to the plexus as used by Koscielnak-Nielsen et al. ${ }^{14}$ These differences also have implications for the validity of results obtained by pooling and meta-analysis of these data.

\section{Appropriate outcome data}

As the place of UGRA in contemporary anesthetic practice changes and grows, investigators need to consider which outcomes are most clinically relevant to their audience. Arguably, the greatest impact of UGRA has been on anesthesiologists who are re-discovering or just learning regional anesthesia. The burning questions for this group include: Will ultrasound help me ensure my blocks are successful? or Will ultrasound help me avoid complications of regional anesthesia? and Which techniques should I choose? The answer to the latter question hinges on more than one outcome. For example, a common obstacle to adopting regional anesthesia is the perceived delay that it introduces into operating room schedules. Therefore, when comparing ultrasound-guided techniques, outcomes such as time to readiness for surgery, which includes image acquisition time, block performance time, and block onset time, are as pertinent as overall block success. At the same time, it must be recognized that statistically significant differences in these indices of time may not be clinically significant. ${ }^{13,14}$

Block success itself is a difficult issue to address, not the least because it is a multi-dimensional outcome. The observed incidence of "block success" or "surgical anesthesia" depends on factors such as the elapsed time between block completion and assessment and the definition of a "block" in terms of quality (e.g., sensory and/or motor numbness and loss of pinprick sensation versus loss 


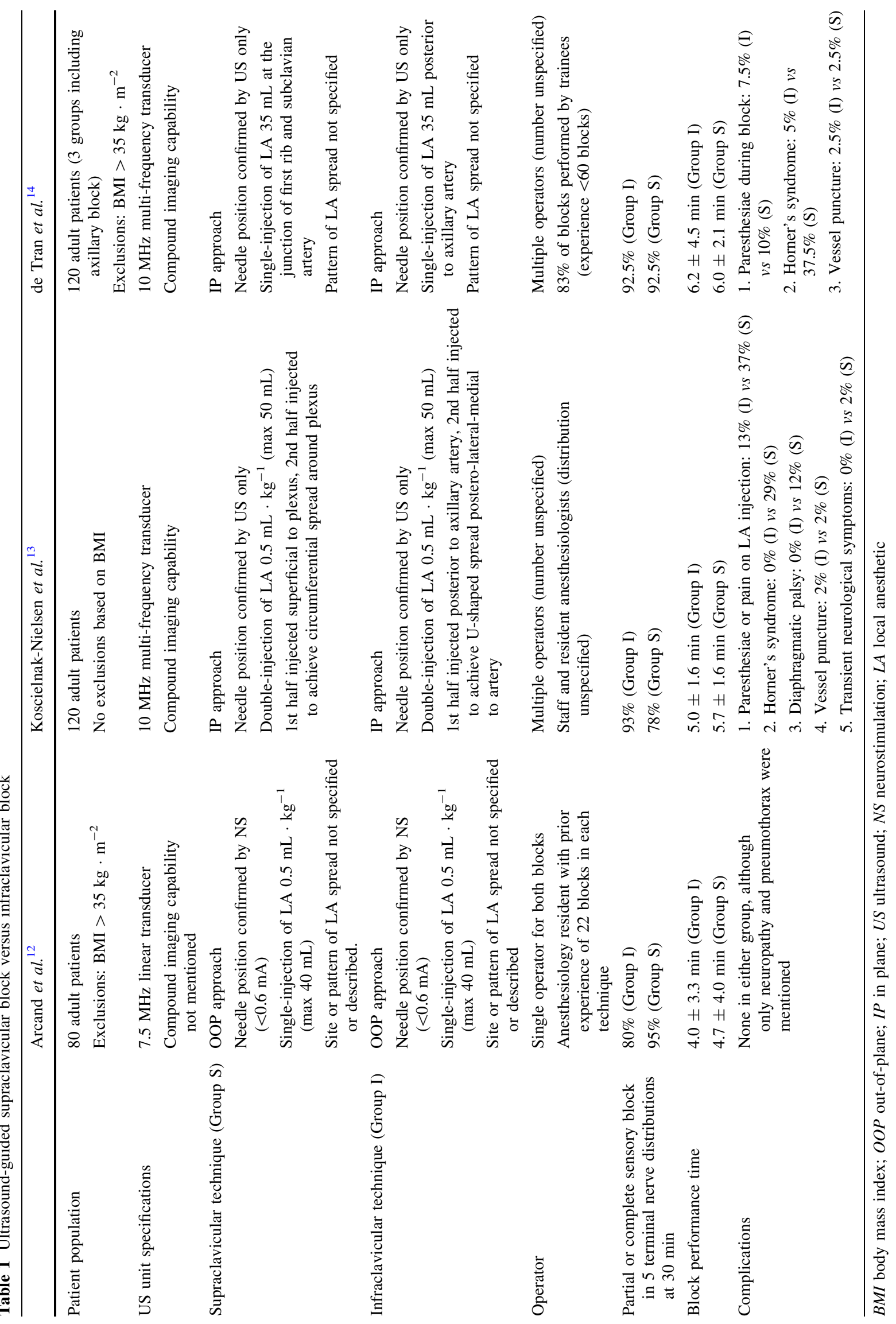


of all sensation) as well as distribution (e.g., all terminal nerve distributions versus site of surgery only). Unfortunately, these factors tend to be inconsistent among studies. Rather than relying on arbitrary definitions of block success, it may be preferable for investigators to report as much objective data as possible, which will allow readers to draw their own conclusions about block efficacy.

"Negative" outcomes, i.e., adverse events, are just as significant as "positive" outcomes. The problem with adverse event reporting in the majority of studies is threefold. First, not all clinically significant adverse events are surveyed. Second, the definition and assessment of these adverse events is often vague and inconsistent. Third, the incidence of serious complications, such as neurological injury, are so low that significant differences are unlikely to be apparent except in a RCT involving tens of thousands of patients. ${ }^{1}$ Ultrasound provides us with unique markers of adverse events, such as intravascular injection (the absence of local anesthetic spread ${ }^{18}$ ) and intraneural injection (expansion of the nerve ${ }^{19}$ ). Sufficient data is lacking to show that these markers translate into a reduction in the incidence of complications.

The issue of underpowered RCTs applies as much to efficacy outcomes as it does to safety outcomes. One solution lies in conducting large multi-center trials. Valuable outcome data may also be obtained from large-scale outcome registries, such as that set up recently by the Australasian Regional Anaesthesia Collaboration (http://www.regional.anaesthesia. org.au). Another option for obtaining data is through systematic review and meta-analysis of the literature. The limitation here is the heterogeneity in definition, assessment, and reporting of outcomes between studies, which at best prevents effective meta-analysis and at worst leads to inappropriate pooling and generation of potentially erroneous results. It should be noted that clinically significant differences in intervention methods, as illustrated by the studies in Table 1, will also diminish the validity of results obtained from meta-analysis.

\section{Conclusion}

We anticipate that ultrasound will be responsible for the renaissance of regional anesthesia within our specialty. As the popularity of UGRA grows, it is imperative that we validate its practice with high-quality, clinically relevant studies. In the future, researchers must begin to look beyond the issues outlined above and towards longer-term patient-oriented outcomes, such as functional recovery after orthopedic surgery ${ }^{20}$ and recurrence or metastasis after cancer surgery. ${ }^{21}$ Only then will UGRA have truly come of age.

\section{L'évaluation des résultats de l'anesthésie régionale échoguidée}

L'échoguidage a révolutionné la pratique de l'anesthésie régionale. L'aspect le plus remarquable de cette révolution est peut-être qu'elle a incité davantage d'anesthésiologistes à intégrer l'anesthésie régionale dans leur pratique. Au cours des dix dernières années, l'anesthésie régionale échoguidée (AREG) s'est développée et est passée d'une technique expérimentale pratiquée par les experts et les enthousiastes à une modalité bien établie, pertinente d'un point de vue clinique et qui a attiré l'attention de la communauté de l'anesthésie générale.

Parallèlement, la littérature traitant de l'AREG a évolué aussi bien en volume qu'en contenu. Une recherche effectuée sur PubMed à l'aide des termes anglais « ultrasound » et " nerve block » a permis d'extraire 32 citations pour l'année 2004, 82 pour 2006, et 158 pour 2008. Les premières publications se rapportaient principalement à des études examinant des techniques de base pour réaliser des blocs nerveux périphériques et l'anatomie échographique pertinente, et étaient en général réalisées chez des volontaires ou de petites cohortes de patients sélectionnés. Aujourd'hui, nous avons atteint le stade où cette base de connaissances est bien établie pour les blocs les plus communs. Les études ultérieures se sont, en toute logique, employées à comparer l'AREG à la technique traditionnelle ou standard utilisée, la neurostimulation. Bien que le débat concernant la supériorité de l'échoguidage par rapport à la neurostimulation batte encore son plein, ${ }^{1}$ il n'en demeure pas moins qu'il est de plus en plus accepté que la capacité de l'échoguidage à donner une vue directe de l'aiguille, du nerf visé et de la distribution de l'anesthésique local est un avantage majeur. Gardons à l'esprit que lors de l'introduction de la neurostimulation, un débat semblable avait fait rage concernant la supériorité de cette technique par rapport à la recherche des paresthésies. ${ }^{2,3} \mathrm{La}$ neurostimulation a petit à petit été acceptée comme la norme de pratique, mais ce changement ne s'est pas fondé sur des études randomisées contrôlées (ERC) de grande échelle et de bonne qualité démontrant de meilleurs résultats; en réalité, les praticiens ont réalisé les avantages que pouvait procurer un critère d'évaluation objectif de la proximité de l'aiguille et du nerf comparativement à un critère subjectif.

C'est pourquoi il est temps d'aller au delà de la question de savoir s'il est mieux d'utiliser la neurostimulation ou l'échoguidage pour l'anesthésie régionale et de nous demander comment intégrer au mieux l'AREG dans la pratique clinique. Malheureusement, les réponses à ces questions ne sont pas claires et sans équivoque dans la 
littérature. Plusieurs facteurs portant à confusion doivent être pris en compte lorsqu'on tente de généraliser les résultats des études publiées et de les rendre applicables à sa propre pratique.

\section{L'expérience de l'opérateur}

Un des facteurs qui portent le plus à confusion lorsqu'on tente d'interpréter les études portant sur l'AREG est lié à la formation et à l'expérience de l'opérateur. Il est évident que, bien que l'échoguidage ait en grande partie démystifié l'art de l'anesthésie régionale, il demeure toutefois une courbe d'apprentissage avant de réaliser une AREG sécuritaire et efficace. ${ }^{4}$ À ce jour, la plupart des études publiées ont été réalisées par quelques praticiens expérimentés, souvent un ou deux seulement par étude. Bien que cette situation soit inévitable pendant l'évolution de toute nouvelle technique, les résultats de ces études pourraient ne pas être généralisables et applicables au nombre croissant d'anesthésiologistes qui en sont à leurs premiers pas avec l'AREG. Les études à venir, et particulièrement des études comparatives, devront tenir compte du biais potentiel qui résulte de l'expérience de l'opérateur avec la technique à l'étude, ou sa préférence pour une technique sur une autre. Une solution serait de comparer deux groupes d'experts, par exemple un groupe ayant exclusivement recours à la neurostimulation à un autre groupe à l'aise en échoguidage. ${ }^{5}$ Une autre possibilité plus faisable serait d'étudier la réalisation d'une AREG par un vaste groupe d'opérateurs sans expérience, comme par exemple une cohorte de résidents en anesthésiologie durant leur stage en anesthésie régionale. Selon nous, les études démontrant les avantages de l'AREG lorsque réalisée par des résidents, comme celles d'Orebaugh (meilleure facilité de réalisation d'un bloc) ${ }^{6}$ et Grau (courbe d'apprentissage plus courte pour l'anesthésie péridurale), ${ }^{7}$ présentent certaines des données probantes les plus convaincantes quant aux avantages de l'AREG parce qu'elles sont le reflet du type d'expérience que les novices auront probablement lorsqu'ils intégreront l'AREG dans leur pratique clinique.

L'American Society of Regional Anesthesia et l'European Society of Regional Anesthesia and Pain Therapy ont récemment publié la première réédition de leurs recommandations conjointes concernant l'éducation et la formation en AREG. ${ }^{8}$ La standardisation de la formation permettra d'aborder la question du biais de l'opérateur dans les études futures. Cependant, il y a encore beaucoup de travail dans ce domaine. La définition de la compétence est encore débattue, et les chercheurs commencent seulement à déterminer les courbes d'apprentissage associées aux diverses techniques d'échoguidage. ${ }^{9}$ Il faut garder à l'esprit que différents blocs par AREG ont différents niveaux de complexité et nécessitent dès lors différents niveaux de compétence. Il faut également déterminer quelles sont les méthodes optimales d'enseignement des compétences requises pour la réalisation d'une AREG; ces méthodes évolueront sans aucun doute de pair avec la mise au point de modèles et de simulateurs plus perfectionnés.

\section{Les variations entre les techniques}

Lorsqu'on analyse la littérature portant sur l'AREG, il est important de saisir les différences subtiles de technique qui pourraient exister entre une étude et une autre, et de déterminer si ces différences pourraient avoir eu un impact sur les résultats. Par exemple, la capacité d'imagerie par ultrason varie d'une machine et d'une étude à l'autre. Les sondes à plus haute résolution et des caractéristiques telles que l'imagerie combinée améliorent considérablement la visualisation des tissus et de l'aiguille, et pourraient également améliorer l'efficacité du bloc. Quelle approche de l'aiguille a été utilisée, dans le champ («in-plane ») ou hors du champ («out-of-plane») de l'ultrason? Quels critères d'évaluation ont été utilisés pour évaluer le contact entre l'aiguille et le nerf et l'injection de l'anesthésique local? Cette question est particulièrement importante étant donné que l'efficacité d'un bloc est probablement influencée par le site et la manière dont l'anesthésique local se dépose, ainsi que par le volume administré. Des études récentes ont évalué le volume minimal nécessaire à réaliser efficacement divers blocs par échoguidage, ${ }^{10,11}$ mais les critères d'évaluation du dépôt de l'anesthésique local associés à des résultats optimaux doivent encore être déterminés. Étant donné que l'architecture des nerfs varie d'un site anatomique à un autre, il est probable que ces critères d'évaluation varieront également d'un bloc à un autre.

Un bon exemple de la complexité de certaines de ces questions émerge lorsqu'on compare trois études examinant des blocs supraclaviculaires et infraclaviculaires réalisés par échoguidage, mais qui parviennent à des résultats très différents (Tableau 1). ${ }^{12-14}$ Arcand et coll. ${ }^{12}$ rapportent une incidence plus élevée d'anesthésie ou d'analgésie dans les cinq territoires des nerfs du plexus brachial lors de l'utilisation d'un bloc supraclaviculaire; dans l'étude de Koscielnak-Nielsen et coll., ${ }^{13}$ le contraire était vrai; Tran et coll. ${ }^{14}$ n'ont quant à eux trouvé aucune différence. Un taux de complications plus élevé avec le bloc supraclaviculaire a également été observé dans deux des études, ${ }^{13,14}$ mais pas dans la troisième. ${ }^{12}$ Une analyse approfondie de ces études suggèrent que les divergences peuvent s'expliquer par des différences de méthodologie des études (par ex. le nombre et l'expérience des opérateurs) et la technique de réalisation du bloc. Par exemple, le 


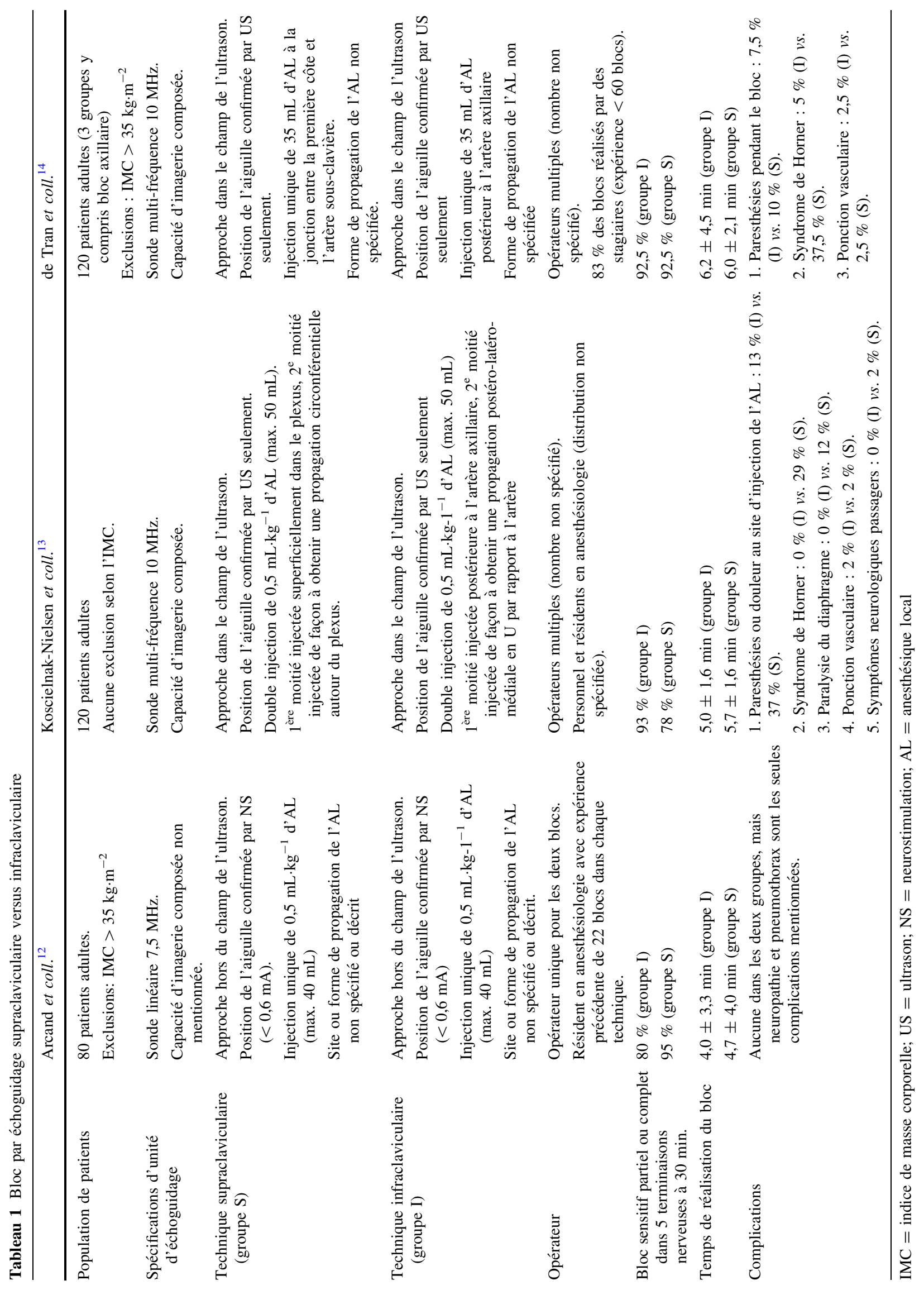


fait qu'Arcand et coll. ${ }^{12}$ n'aient pas spécifié une distribution postéro-latéro-médiale en U de l'anesthésique local en tant que critère d'évaluation du bloc infraclaviculaire pourrait avoir joué un rôle dans le taux de réussite observé plus bas. ${ }^{15}$ De même, une technique optimale de bloc supraclaviculaire peut impliquer une injection profonde d'anesthésique local dans le plexus à la jonction de la première côte et de l'artère sous-clavière ${ }^{16,17}$ (comme dans l'étude de Tran et coll. ${ }^{14}$ ) plutôt qu'une injection superficielle dans le plexus (comme dans l'étude de KoscielnakNielsen et coll. ${ }^{14}$ ). Ces différences ont également des répercussions sur la validité des résultats obtenus lorsqu'on regroupe ces données et qu'on effectue une méta-analyse.

\section{Des données de résultats adaptées}

La place occupée par l'AREG dans la pratique contemporaine de l'anesthésie évolue et grandit; pour cette raison, les chercheurs doivent réfléchir à la pertinence clinique de leurs résultats pour leur public. Le plus grand impact de l'AREG s'est sans doute fait sentir chez les anesthésiologistes qui redécouvrent ou qui apprennent l'anesthésie régionale. "L'échoguidage m'aidera-t-il à assurer la réussite de mes blocs? » «L'échoguidage m'aidera-t-il à prévenir les complications liées à l'anesthésie régionale? » «Quelle technique devrais-je utiliser?» Voici certaines des questions brûlantes pour ce groupe de praticiens. La réponse à la dernière question dépend de plus d'un critère. Par exemple, une des réticences fréquentes à l'adoption de l'anesthésie régionale est liée au délai perçu que cette technique implique dans les horaires de salle d'opération. Par conséquent, lors de la comparaison de différentes techniques d'échoguidage, des critères d'évaluation tels que le temps de préparation jusqu'à la chirurgie (qui comprend le temps d'acquisition des images, le temps de réalisation du bloc et le délai jusqu'à efficacité du bloc) sont tout aussi importants que le succès global du bloc. Néanmoins, il faut garder à l'esprit que des différences statistiquement significatives de ces indices de temps pourraient ne pas l'être d'un point de vue clinique. ${ }^{13,14}$

La réussite d'un bloc est en soi une question difficile à aborder, particulièrement étant donné qu'il s'agit d'un résultat à plusieurs facettes. L'incidence observée de «succès d'un bloc » ou «d'anesthésie chirurgicale » dépend de facteurs tels que le temps écoulé entre la réalisation du bloc et son évaluation, ainsi que de la définition d'un «bloc » en termes de qualité (par ex. sensoriel ou moteur ou les deux, perte de sensation de piqûre d'aiguille par rapport à perte de toute sensation) et de sa distribution (par ex. les territoires de tous les nerfs ou le site chirurgical seulement). Malheureusement, ces facteurs ont tendance à ne pas être identiques d'une étude à une autre. Plutôt que de se fonder sur des définitions arbitraires pour déterminer le succès d'un bloc, il serait préférable que les chercheurs rapportent autant de données objectives que possible, afin de permettre au lecteur de tirer ses propres conclusions quant à l'efficacité d'un bloc.

Les résultats « négatifs », comme les effets secondaires néfastes, sont tout aussi importants que les résultats « positifs ». Dans la majorité des études, le problème de déclaration des effets secondaires négatifs est triple. Premièrement, les effets secondaires négatifs significatifs d'un point de vue clinique ne sont pas tous évalués. Deuxièmement, la définition et l'évaluation de ces effets secondaires négatifs sont souvent vagues et contradictoires. Troisièmement, l'incidence de complications graves telles que les lésions neurologiques est tellement basse qu'il est improbable que des différences significatives apparaissent, sauf dans une étude randomisée contrôlée (ERC) portant sur des dizaines de milliers de patients. ${ }^{1}$ L'échoguidage nous procure des marqueurs uniques d'effets secondaires néfastes, comme par exemple l'injection intravasculaire (absence de propagation de l'anesthésique $\operatorname{local}^{18}$ ) et l'injection intraneurale (expansion du nerf ${ }^{19}$ ). Ce qui nous fait défaut, ce sont des données suffisantes pour montrer que ceci se traduit par une réduction de l'incidence de complications.

Le problème des ERC à trop faible puissance s'applique autant aux résultats concernant l'efficacité qu'à ceux portant sur la sécurité. Une des solutions serait de mener des études multicentriques d'envergure. Des données précieuses concernant les résultats pourraient également être extraites de registres de devenirs à grande échelle, tel que celui récemment créé par l'Australasian Regional Anaesthesia Collaboration (http://www.regional.anaesthesia.org. au). Une autre possibilité pour obtenir des données consisterait à effectuer une revue méthodique et une métaanalyse de la littérature. En revanche, une telle méthode comporte des limites en raison de l'hétérogénéité des définitions, de l'évaluation et de la formulation des résultats entre les études. Dans le meilleur des cas, cet état de fait entrave une méta-analyse efficace; dans le pire, cela mène à un regroupement inadéquat des résultats et à la génération de résultats potentiellement erronés. Il faut noter que les différences significatives d'un point de vue clinique dans les méthodes d'intervention, telles qu'illustrées dans le Tableau 1, réduiront également la validité des résultats obtenus lors d'une méta-analyse.

\section{Conclusion}

Selon nous, l'échoguidage sera le fer de lance de la renaissance de l'anesthésie régionale dans notre spécialité. Alors que grandit la popularité de l'AREG, il est essentiel 
de valider sa pratique à l'aide d'études de haute qualité et pertinentes d'un point de vue clinique. À l'avenir, les chercheurs devront également aller au delà des questions émises ci-dessus et se tourner vers des résultats plus pertinents pour les patients et sur le plus long terme, comme par exemple la récupération fonctionnelle après une chirurgie orthopédique, ${ }^{20}$ et la récidive ou la métastase après une chirurgie oncologique. ${ }^{21} \mathrm{C}$ 'est à ce moment seulement que l'AREG atteindra sa majorité.

Disclosures Dr. Vincent Chan receives equipment support for research from Philips Medical Systems, GE Healthcare and SonoSite.

Competing interests None declared.

\section{References}

1. Borgeat A, Capdevila X. Neurostimulation/ultrasonography: the Trojan war will not take place. Anesthesiology 2007; 106: 896-8.

2. Moore DC. "No paresthesias-no anesthesia", the nerve stimulator or neither? Reg Anesth 1997; 22: 388-90.

3. VadeBoncouer TR, Riegler FX. In defense of the nerve stimulator. Reg Anesth Pain Med 1998; 23: 229-30.

4. Sites BD, Spence BC, Gallagher JD, Wiley CW, Bertrand $M L$, Blike GT. Characterizing novice behavior associated with learning ultrasound-guided peripheral regional anesthesia. Reg Anesth Pain Med 2007; 32: 107-15.

5. van Geffen GJ, van den Broek E, Braak GJ, Giele JL, Gielen MJ, Scheffer GJ. A prospective randomised controlled trial of ultrasound guided versus nerve stimulation guided distal sciatic nerve block at the popliteal fossa. Anaesth Intensive Care 2009; 37: 32-7.

6. Orebaugh SL, Williams BA, Kentor $M L$. Ultrasound guidance with nerve stimulation reduces the time necessary for resident peripheral nerve blockade. Reg Anesth Pain Med 2007; 32: 448-54.

7. Grau T, Bartusseck E, Conradi R, Martin E, Motsch J. Ultrasound imaging improves learning curves in obstetric epidural anesthesia: a preliminary study. Can J Anesth 2003; 50: 1047-50.

8. Sites BD, Chan VW, Neal JM, et al. The American Society of Regional Anesthesia and Pain Medicine and the European Society of Regional Anaesthesia and Pain Therapy Joint Committee recommendations for education and training in ultrasound-guided regional anesthesia. Reg Anesth Pain Med 2009; 34: 40-6.

9. Helayel PE, Conceicao DB, Nascimento BS, Kohler A, Boos GL, Oliveira Filho GR. Learning curve for the ultrasound anatomy of the brachial plexus in the axillary region. Rev Bras Anestesiol 2009; 59: 187-93.

10. Duggan E, El Beheiry H, Perlas A, et al. Minimum effective volume of local anesthetic for ultrasound-guided supraclavicular brachial plexus block. Reg Anesth Pain Med 2009; 34: 215-8.

11. O'Donnell BD, Iohom $G$. An estimation of the minimum effective anesthetic volume of $2 \%$ lidocaine in ultrasound-guided axillary brachial plexus block. Anesthesiology 2009; 111: 25-9.

12. Arcand $G$, Williams SR, Chouinard $P$, et al. Ultrasound-guided infraclavicular versus supraclavicular block. Anesth Analg 2005; 101: 886-90.

13. Koscielniak-Nielsen ZJ, Frederiksen BS, Rasmussen H, Hesselbjerg $L$. A comparison of ultrasound-guided supraclavicular and infraclavicular blocks for upper extremity surgery. Acta Anaesthesiol Scand 2009; 53: 620-6.

14. de Tran QH, Russo G, Munoz L, Zaouter C, Finlayson RJ. A prospective, randomized comparison between ultrasound-guided supraclavicular, infraclavicular, and axillary brachial plexus blocks. Reg Anesth Pain Med 2009; 34: 366-71.

15. Bloc S, Garnier T, Komly B, et al. Spread of injectate associated with radial or median nerve-type motor response during infraclavicular brachial-plexus block: an ultrasound evaluation. Reg Anesth Pain Med 2007; 32: 130-5.

16. Soares $L G$, Brull R, Lai J, Chan VW. Eight ball, corner pocket: the optimal needle position for ultrasound-guided supraclavicular block. Reg Anesth Pain Med 2007; 32: 94-5.

17. de Tran QH, Munoz L, Russo G, Finlayson RJ. A trick shot to the corner pocket. Reg Anesth Pain Med 2008; 33: 503-4.

18. Brull R, Perlas A, Cheng PH, Chan VW. Minimizing the risk of intravascular injection during ultrasound-guided peripheral nerve blockade. Anesthesiology 2008; 109: 1142.

19. Chan VW, Brull R, McCartney CJ, Xu D, Abbas S, Shannon P. An ultrasonographic and histological study of intraneural injection and electrical stimulation in pigs. Anesth Analg 2007; 104: 1281-4.

20. Shum CF, Lo NN, Yeo SJ, Yang KY, Chong HC, Yeo SN. Continuous femoral nerve block in total knee arthroplasty: immediate and two-year outcomes. J Arthroplasty 2009; 24: 204-9.

21. Exadaktylos AK, Buggy DJ, Moriarty DC, Mascha E, Sessler DI. Can anesthetic technique for primary breast cancer surgery affect recurrence or metastasis? Anesthesiology 2006; 105: 660-4. 